Research Paper

\title{
Logarithmic bacterial gradient chip for analyzing the effects of dietary restriction on $C$. elegans growth
}

\author{
Anle $\mathrm{Ge}^{\mathrm{a}}$, Liang $\mathrm{Hu}^{\mathrm{a}}$, Xixian Wang ${ }^{\mathrm{a}, \mathrm{b}}$, Jinchi Zhu ${ }^{\mathrm{a}}$, Xiaojun Feng ${ }^{\mathrm{a}}$, Wei Du ${ }^{\mathrm{a}, *}$, Bi-Feng Liu ${ }^{\mathrm{a}}$ \\ a The Key Laboratory for Biomedical Photonics of MOE at Wuhan National Laboratory for Optoelectronics, Hubei Bioinformatics E Molecular Imaging Key \\ Laboratory, Systems Biology Theme, Department of Biomedical Engineering, College of Life Science and Technology, Huazhong University of Science and \\ Technology, Wuhan 430074, China \\ ${ }^{\mathrm{b}}$ Single Cell Center, CAS Key Laboratory of Biofuels and Shandong Key Laboratory of Energy Genetics, Qingdao Institute of Bioenergy and Bioprocess \\ Technology, Chinese Academy of Sciences, Qingdao, Shandong, 266101, China
}

\section{A R T I C L E I N F O}

\section{Article history:}

Received 17 February 2017

Received in revised form 4 August 2017

Accepted 9 August 2017

Available online 14 August 2017

\section{Keywords:}

Microfluidic device

Caenorhabditis elegans

Logarithmic bacterial gradient

Dietary restriction

Lifespan extension

\begin{abstract}
A B S T R A C T
C. elegans is a commonly used model organism for studying the basic mechanisms of lifespan extension by dietary restriction (DR). Progress has been limited, however, by the lack of an automated system for quantitative analysis of this effect of bacterial diet. In this work, multifunctional logarithmic gradientcustomizing microfluidic device, which takes advantage of hydrodynamics, was developed to long-term maintain worm culture on the chip with parallel live imaging and generate logarithmic concentration gradient of bacteria. We successfully employed this platform to investigate the longevity and stress response of the worms in response to various concentrations of bacterial food. The results indicated that the lifespan extension of the worms was induced by DR when bacteria density was $10^{8}$ cells mL $\mathrm{mL}^{-1}$ and further dilutions would reduce the lifespan, which proposed a new dietary restriction regime in $C$. elegans. In addition, imaging analysis showed that DR regulated the subcellular localization DAF-16 in a way different from starvation that reduced lifespan of the worms. The platform allows not only horizontal studies of $C$. elegans in response to various environments over a wide range of concentrations, but also multiparameter evaluation of longevity in the worms with high temporal resolution, providing clues to better understand the aging process and help age-related drug screen.
\end{abstract}

(c) 2017 Elsevier B.V. All rights reserved.

\section{Introduction}

Dietary restriction (DR) can retard aging and increase lifespan in a wide range of species, including yeast, worms, flies, and mammals [1]. Evidences indicate that DR results in several physiological changes, such as increased autophagy, increased resistance to a variety of stresses, decreased response to nutrient and growth factor, which contribute to its effects on longevity [2,3]. Thus, DR is most commonly used method of retarding senescence and reducing mortality at present. Understanding the mechanism underlying this environmental intervention on lifespan is of vital importance $[4,5]$. Although numerous hypotheses have been proposed to explain the extension of DR to longevity, the exact mechanisms of regulatory role of DR in aging and lifespan is still poorly understood due to technical difficulties in precise dietary control.

\footnotetext{
* Corresponding author at: College of Life Science and Technology, Huazhong University of Science and Technology, Wuhan 430074, China

E-mail address: weidu@mail.hust.edu.cn (W. Du).
}

The nematode Caenorhabditis elegans (C. elegans) has been a primary model organism for studying basic mechanisms of aging and longevity physiology, because of its fully sequenced genome, short generation time $\left(\sim 3.5\right.$ days at $\left.20^{\circ} \mathrm{C}\right)$ and lifespan $(2-3$ weeks) [6,7]. Several aging-related factors firstly uncovered from studies in $C$. elegans, such as the insulin/IGF-1 signaling pathway and the target of rapamycin kinase, are now well-known to similarly modulate longevity and aging in mammals [8]. In addition, the worms' stress response to diverse environmental cues also plays a similar role in adjusting lifespan in mammals. Therefore, understanding the mechanisms responsible for the worms' response to DR is scientifically interesting and important.

Currently, researchers mainly use genetic and environmental manipulations to model DR condition to study the effect of food consumption on lifespan in C. elegans $[7,9,10]$. In the traditional methods, mutant worms (such as eat-2) with decreased feeding rate is used for genetically mimicking the worms treated with DR $[11,12]$. However, this genetic mimic could have pleiotropic effects unrelated to DR, which may interfere with experimental outcomes. Another method of dilution of the worms' food was also used for 
mimicking DR, in which the worms usually were maintained on nematode growth medium (NGM) agar plates seeded with a few growth-arrested bacteria. To screen optimum concentration of bacteria food for modeling DR condition, large number of agar plates containing varying concentration of bacteria are needed. However, these traditional methods suffer from several disadvantages for obtaining reliable data. First of all, these methods are rather timeconsuming due to stepwise dilution of bacteria and transfer of the worms to new plates every 1-2 days. In addition, the concentration of bacteria food on agar plate during whole experiments could not be stably maintained because of the consumption by the worms, which make it difficult to compare the data from different laboratories due to inaccurate food control. These agar plate-based methods also lack the capability of investigating physiological changes of the worms in real time, such as chemical stimulation and imaging at high resolution. Although important findings have been achieved using these DR regimens in C. elegans, it is necessary to develop a more efficient and harmless method for modeling DR condition.

Microfluidic device have been proven useful for the biochemical and biological experiments, featuring good transparency, gas permeability, biocompatibility and high throughput [13-17]. In recent years, microfluidic device has been recognized as a new platform for fundamental studies in C. elegans because of microchannel's comparable dimension to the tiny worms and the versatile use of micro-flow. "Worm-chip" have successfully used in phenotyping and stage screening, nerve system imaging, behavioral dynamics, microsurgery and microinjection [18-27]. Currently, several dilution micro-devices have been proposed for generating logarithmic, liner, and parabolic chemical concentration gradients [28-36]. Compared to traditional methods, microfluidic devices could generate linear chemical concentration gradient which shown great potential in studying chemotaxis behavior [22,37-39], screening of chemotaxis-defective mutants $[23,40]$ and drug screening in C. elegans $[20,37,41]$. Although these "Christmas tree" mixers had been well established and applied to create concentration gradients, they are not suitable for generating wide-range concentration gradient, such as 1000 fold dilution. The establishment of wide-range bacterial concentration gradient plays essential roles in screening optimal food condition for modeling DR treatment in C. elegans. However, there is currently no report on the effect of food concentration on the lifespan of $C$. elegans in microfluidic device due to difficulties in generating large concentrations range and diluting the bacteria with a low diffusion coefficient. Microfluidic-based DR condition modeling in C. elegans should partially automate the studies of DR's extension of the lifespan, which would be a versatile tool for worm researchers.

To systematically analyze the short-term and long-term effects of bacterial food over a wide range of concentrations, we developed a microfluidic device for establishing on-chip logarithmic concentration gradient of bacteria, long-term worm culture, and parallel live imaging. Gradient of bacterial food supplements with four orders of magnitude were automatically generated by simply perfusing two streams into our device. We employed this platform to evaluate the effects of a large range of bacteria concentration on the lifespan of C. elegans. In addition, DAF-16:GFP nuclear localization induced by various concentrations of bacterial food were also investigated to better understand the mechanism of dietary restriction's effect on the worms.

\section{Materials and methods}

\subsection{Chemicals and reagents}

Reagents of analytical grade including $\mathrm{CaCl}_{2}, \mathrm{NaCl}, \mathrm{Na}_{2} \mathrm{HPO}_{4}$, $\mathrm{KH}_{2} \mathrm{PO}_{4}, \mathrm{~K}_{2} \mathrm{HPO}_{4}, \mathrm{MgSO}_{4}$, and ethanol were purchased from
Sinopharm Chemical Reagent (Shanghai, China). Pluronic F-127, fluorescein sodium salt, peptone, tryptone, yeast extract and cholesterin were purchased from Sigma-Aldrich (St. Louis, MO, USA). All regents used in our experiment were of analytical grade. GM 1070 SU-8 negative photoresist was purchased from Gersteltec (Pully, Switzerland). Polydimethylsiloxane (PDMS Sylgard 184) and curing agent (Sylgard 184) were purchased from Dow Corning (Midland MI USA). All solutions were prepared with water purified by PURIST ${ }^{\circledR}$ Systems (RephiLe, Shanghai, China) and were sterilized by filtration $(0.22 \mu \mathrm{m}$ microporous membrane filtration) or by using an autoclave $\left(121^{\circ} \mathrm{C}, 30 \mathrm{~min}\right)$ prior to use. M9 buffer was obtained by adding $3 \mathrm{~g} \mathrm{KH} \mathrm{PO}_{4}, 6 \mathrm{~g} \mathrm{Na} \mathrm{HPO}_{4}, 5 \mathrm{~g} \mathrm{NaCl}$ and $1 \mathrm{~mL}$ $\mathrm{MgSO}_{4}(1 \mathrm{M})$ to 1 Liter $\mathrm{H}_{2} \mathrm{O}$. Pluronic F-127 solution was prepared by diluting 5\% (weight/volume) Pluronic F-127 in M9 buffer.

\subsection{Worm culture and strains}

A single E. coli (OP50) colony was inoculated into $25 \mathrm{~mL}$ of LB medium (10.0 g of tryptone, $5.0 \mathrm{~g}$ of yeast extract and $5.0 \mathrm{~g}$ of $\mathrm{NaCl}$ per liter of distilled water) and shaken overnight at $37^{\circ} \mathrm{C}$. The saturated bacteria solution was stored at $4{ }^{\circ} \mathrm{C}$ as the food. C. elegans were cultured at $24^{\circ} \mathrm{C}$ in NGM agar plate seeded with OP50. For all experiments, the worms of wild-type Bristol N2 and transgenic strain TJ356 [zIs356 IsPdaf-16:daf-16-gfp; rol-6(su1006)] expressing a DAF-16:GFP fusion protein in cells were used. These strains were obtained from the Caenorhabditis Genetics Centre (CGC) at the University of Minnesota (St. Paul). The worms were firstly synchronized prior to assay for ensuring experimental consistency. Briefly, five young gravid worms were placed on a NGM agar plate seeded with OP50 and allowed to lay eggs for $4 \mathrm{~h}$. Then these synchronized eggs were cultured for 2.5 days under standard conditions until the worms reached the young adult stage.

\subsection{Fabrication of microfluidic device}

We fabricated this microfluidic device with two-layer PDMS structure. As shown in Fig. 1, the top layer (colored red, $5 \mathrm{~mm}$ in thick) containing microchannels ( $25 \mu \mathrm{m}$ in height) for controlling the status of the microchannel in the bottom. The bottom layer (colored blue, $100 \mu \mathrm{m}$ in thick) containing a PDMS membrane $(20 \mu \mathrm{m}$ in thick) on the top of microchannels with a height of $80 \mu \mathrm{m}$ for worm culture, food delivery and concentration gradient generation. The microchannels for food delivery and gradient generation were $200 \mu \mathrm{m}$ in width. At the end of worm inlet channel there were some narrow wedge structures with from 100 to $25 \mu \mathrm{m}$ in width. The chambers for worm culture were designed with a dimension of $5 \mathrm{~mm}$ in length and $0.5 \mathrm{~mm}$ in width (c).

Both layers of PDMS structure were fabricated according to standard soft lithography and rapid prototyping. Briefly, the bottom and top layer microchannels were first patterned on two different silicon wafers by spinning photoresist SU-8 ${ }^{\mathrm{TM}}$ at heights of $80 \mu \mathrm{m}$ and $20 \mu \mathrm{m}$, respectively. The PDMS with mixing ratio of 10:1 was poured onto the top SU-8 masters to create a top PDMS layer with high mechanical integrity as usual. The PDMS with mixing ratio of 20:1 was poured onto the bottom silicon wafer pre-treated with trimethylchlorosilane vapor, and then spun at $1200 \mathrm{rpm}$ on a spin coater to fabricate the bottom PDMS membrane with large membrane deflections. After curing at $65^{\circ} \mathrm{C}$ for $4 \mathrm{~h}$ in an oven, these two PDMS layers were aligned and irreversibly bonded together by oxygen plasma treatment, forming a thin PDMS membrane between top valves and bottom channels. Finally, the bonded PDMS layer was peeled off from the silicon substrate and bonded to a glass coverslip treated by oxygen plasma to form the final device. 

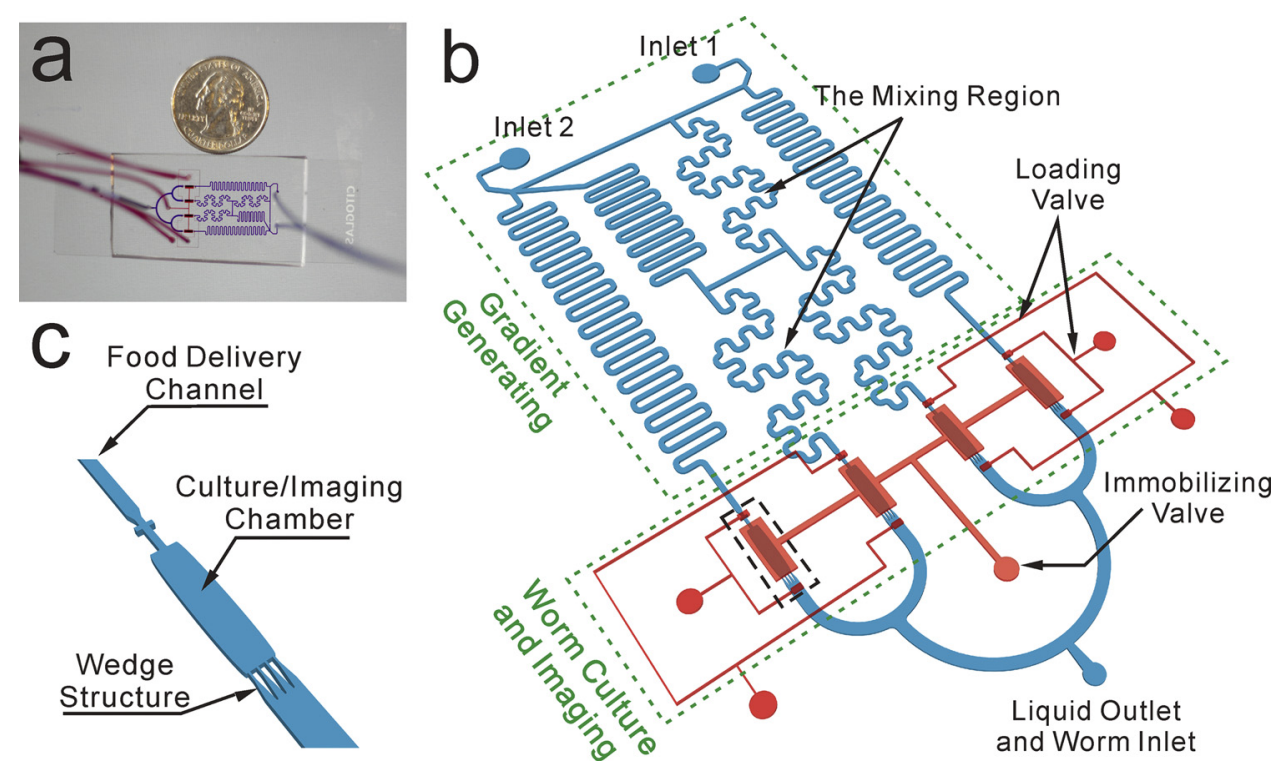

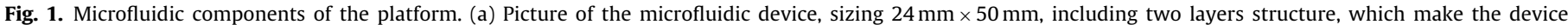

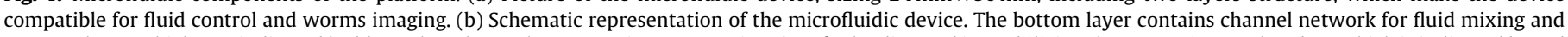

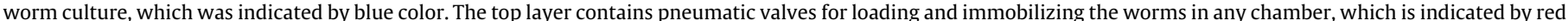

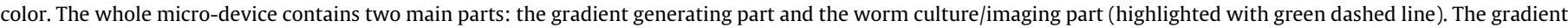

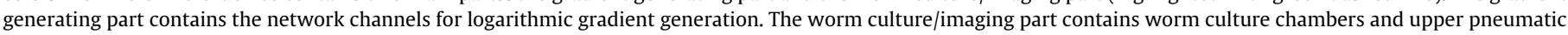

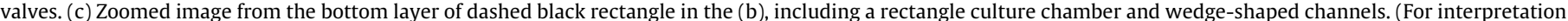
of the references to colour in this figure legend, the reader is referred to the web version of this article.)

\subsection{Generation of logarithmic gradient of bacteria}

The design of the micro-device is based on the adjustment of volumetric flow rate of bacteria solution due to the theory and mathematic model for microfluidic networks [32-34], which was simply determined by the length of the distribution channels. Two bacteria stock solutions with different initial concentration were delivered by the distribution channels under a constant positive pressure to obtain 10 -fold serial dilution. Since the microscale flow was laminar in the channels due to the low Reynolds number, the pressure drop could be described by the Hagen-Poiseuille equation:

$\Delta p=\frac{8 \eta L Q}{\pi R^{4}}$

$\Delta p=Q R_{f}$

Where $\Delta p, \eta, L, Q$ and $R$ represent the pressure drop, the dynamic viscosity of the fluid, the length of the microchannel, the volumetric flow rate and the effective radius of the microchannel, respectively. $R_{f}$ represent the hydrodynamic resistance of the channel. Assuming that the volumetric flow rate remains constant for the laminar flows, $R_{f}$ can be described as following:

$R_{f}=\frac{8 \eta L}{\pi R^{4}}$

In out device, the cross-sections of the channels are fixed. And the dynamic viscosity of the fluid remains consistent. Thus, the hydrodynamic resistance of the channel $R_{F}$ is proportional to the length of the channel, which can be presented as: $R_{F} \propto L$.

As shown in Fig. 2, based on Kirchhoff's current law, the parameter of flow will be satisfied as the following:

$\mathrm{Q}_{\mathrm{L}}=\mathrm{Q}_{0}+\mathrm{Q}_{\mathrm{L}, 1}+\mathrm{Q}_{\mathrm{L}, 2}$

$\mathrm{Q}_{\mathrm{R}}=\mathrm{Q}_{3}+\mathrm{Q}_{\mathrm{R}, 2}$

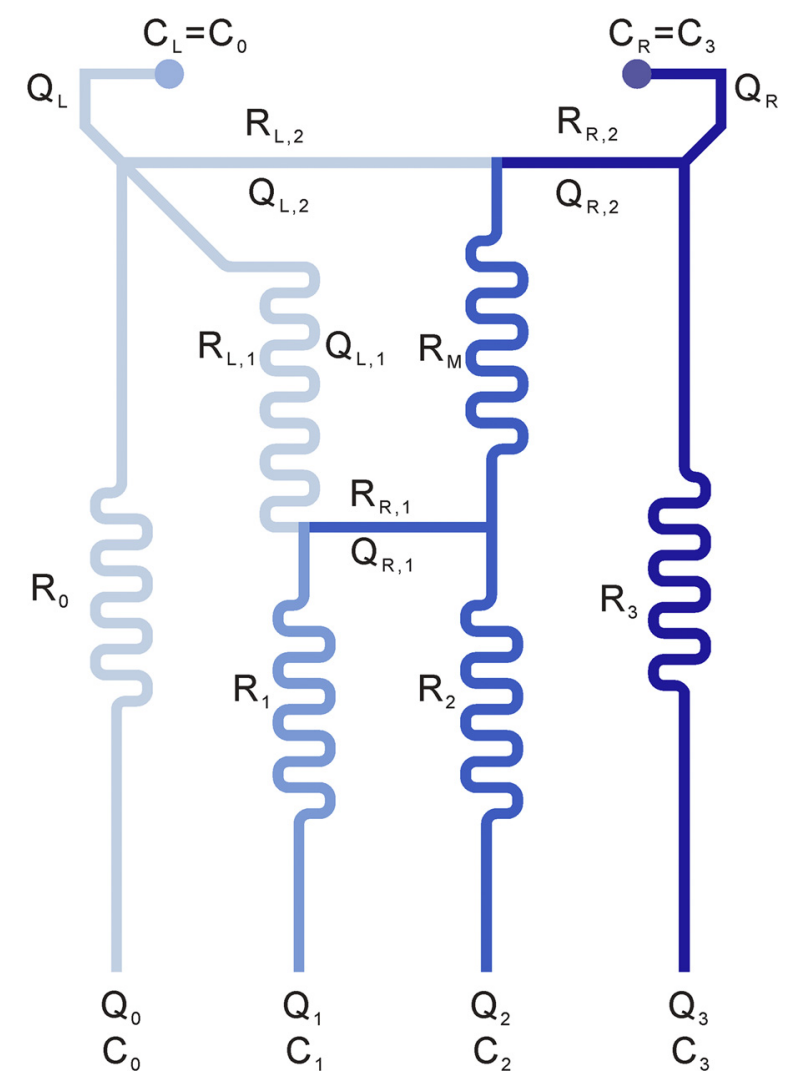

Fig. 2. The mathematical modeling of the microfluidic circuit network generating a 10 -fold log serial dilution. Details of each parameter are presented in appropriate place. 
Table 1

The parameters for 10 -fold logarithmic gradient chip.

\begin{tabular}{|c|c|c|c|}
\hline Flow resistance & Function expression & Expression by $R_{M}, R_{S}$ & Experimental data \\
\hline $\mathrm{R}_{0}$ & $R_{L, 1} \frac{Q_{L, 1}}{Q_{0}}+R_{1} \frac{Q_{1}}{Q_{0}}$ & $1.09 \mathrm{R}_{\mathrm{S}}+2.1 \mathrm{R}_{\mathrm{M}}$ & 66.92 \\
\hline $\mathrm{R}_{1}$ & $\mathrm{R}_{\mathrm{M}, 1}$ & $\mathrm{R}_{\mathrm{M}}$ & 30 \\
\hline $\mathrm{R}_{2}$ & $\left(R_{S} \frac{C_{1}-C_{0}}{C_{2-} C_{0}}+R_{M}\right) \frac{Q_{1}}{Q_{2}}$ & $\frac{1}{11} R_{S}+R_{M}$ & 30.33 \\
\hline $\mathrm{R}_{3}$ & $R_{S} \frac{Q_{R, 2}}{Q_{3}}+R_{M} \frac{Q_{L, 2+} Q_{R, 2}}{Q_{3}}+R_{2} \frac{Q_{2}}{Q_{3}}$ & $0.2 R_{S}+2.1 R_{M}$ & 63.72 \\
\hline $\mathrm{R}_{\mathrm{L}, 1}$ & $R_{L, 2} \frac{Q_{L, 2}}{Q_{L, 1}}+R_{M} \frac{Q_{L, 2}+Q_{R, 2}}{Q_{L, 1}}+R_{S} \frac{Q_{R, 1}}{Q_{L, 1}}$ & $1.211 \mathrm{R}_{\mathrm{S}}+1.222 \mathrm{R}_{\mathrm{M}}$ & 40.96 \\
\hline $\mathrm{R}_{\mathrm{L}, 2}$ & $2 R_{S}$ & $2 \mathrm{R}_{S}$ & 7.2 \\
\hline $\mathrm{R}_{\mathrm{R}, 1}$ & $\mathrm{R}_{\mathrm{S}}$ & $\mathrm{R}_{\mathrm{S}}$ & 3.6 \\
\hline $\mathrm{R}_{\mathrm{R}, 2}$ & $\mathrm{R}_{\mathrm{S}}$ & $\mathrm{R}_{\mathrm{S}}$ & 3.6 \\
\hline
\end{tabular}

$\mathrm{Q}_{\mathrm{L}}+\mathrm{Q}_{\mathrm{R}}=\mathrm{Q}_{0}+\mathrm{Q}_{1}+\mathrm{Q}_{2}+\mathrm{Q}_{3}$

$\mathrm{Q}_{0}=\mathrm{Q}_{\mathrm{L}}-\mathrm{Q}_{\mathrm{L}, 1}-\mathrm{Q}_{\mathrm{L}, 2}$

$\mathrm{Q}_{1}=\mathrm{Q}_{\mathrm{L}, 1}+\mathrm{Q}_{\mathrm{R}, 1}$

$\mathrm{Q}_{2}=\mathrm{Q}_{\mathrm{L}, 2}+\mathrm{Q}_{\mathrm{R}, 2}-\mathrm{Q}_{\mathrm{R}, 1}$

$\mathrm{Q}_{3}=\mathrm{Q}_{\mathrm{R}}-\mathrm{Q}_{\mathrm{R}, 2}$, where $\mathrm{Q}_{\mathrm{R}, 0}=$ 0and $_{0}=\mathrm{Q}_{1}=\mathrm{Q}_{2}=\mathrm{Q}_{3}$

To achieve the 10 -fold logarithmic concentration gradient with $\left(C_{0}, C_{1}, C_{2}, C_{3}\right)=(0.1,1,10,100 \%)$, the mixing ratios $\left(Q_{L, 1}: Q_{R, 1}, Q_{L, 2}\right.$ : $\left.\mathrm{Q}_{R, 2}\right)$ were $(9: 1,9: 1)$ respectively. Under the given conditions we can deduce the formula of mixing part containing $Q_{0}$ :

$\mathrm{Q}_{\mathrm{L}, 1}=0.9 \mathrm{Q}_{1}=0.9 \mathrm{Q}_{0}$

$\mathrm{Q}_{\mathrm{R}, 1}=0.1 \mathrm{Q}_{1}=0.1 \mathrm{Q}_{0}$

$\mathrm{Q}_{\mathrm{L}, 2}=0.9\left(\mathrm{Q}_{1}+0.1 \mathrm{Q}_{2}\right)=0.99 \mathrm{Q}_{0}$

$\mathrm{Q}_{\mathrm{R}, 2}=0.1\left(\mathrm{Q}_{1}+0.1 \mathrm{Q}_{2}\right)=0.11 \mathrm{Q}_{0}$

Simplifying the analysis and design of the circuits, the following set of design rules for the flow resistances of mixing and serial cascading channels can be applied. $R_{M, 1}=R_{M, 2}=R_{M}, R_{R, 1}=R_{R, 2}=R_{S}$, $R_{L, 2}=2 R_{S}$. Where $R_{M}$ is the flow resistance of the mixing channels and $R_{S}$ is the flow resistance of the serial-cascading channels. The flow resistance $R_{M}$ of the mixing channels can be determined based on the mixing performance of solution. $R_{S}$ can be determined based on the chip total width (total width equal to $3 R_{S}$ ).

The flow resistances of each channel can be determined using Kirchhoff's voltage law. We can calculate other flow resistances with known parameters include $\mathrm{R}_{\mathrm{M}}$ and $\mathrm{R}_{\mathrm{S}}$. During the experiment, the $R_{M}=30 \mathrm{~mm}$ and the $R_{S}=3.6 \mathrm{~mm}$. The dimensions of each channel are provided in Table 1 . To sum up, by adjusting the flow rate of inlet 1 and inlet 2 to $1: 2.6$, a bacteria concentration gradient of $1: 10: 100: 1000$ can be obtained in the four channels.

\subsection{COMSOL multiphysics simulation}

The system was modeled as steady state using the incompressible Navier-Stokes module. The dilution efficiency was validated using a 3D finite volume model. The properties of fluids for the simulation were Newtonian with density $10^{3} \mathrm{~kg} \mathrm{~m}^{-3}$, viscosity $10^{-3}$ Pa s and diffusion coefficient $4.9 \times 10^{-10} \mathrm{~m}^{2} \mathrm{~s}^{-1}$ (fluorescein in water). The height of model was set as $80 \mu \mathrm{m}$. For the inlets, one stock solution was the pure water, the other was simulated solute. To demonstrate the dilution ratio of different channels, a stock solution ( $1000 \%, 0 \%$ oquals to water) with a flow rate of $1 \mu \mathrm{L} \mathrm{min}^{-1}$ was applied in inlet 1 and a dilute solution (water) with a flow rate of $2.6 \mu \mathrm{L} \mathrm{min}^{-1}$ was applied in inlet 2 . The criterion for convergence was set for the increment in each variable to fall below $1 \times 10^{-5}$. A mass flow inlet condition was used for the inlet 1 and inlet 2 . A zero pressure boundary condition was set for the outlet.

\subsection{Worms loading and immobilization on chip}

As shown in Fig. 3a and Video S1, the worms suspended in the M9 buffer were introduced into the microfluidic device through Teflon tubes $(0.7 \mathrm{~mm}$ in inside diameter and $1.2 \mathrm{~mm}$ in outside diameter) using a hand-hold syringe. Firstly, the micro-valves for worm loading in the top layer were opened for injecting the worms into the chamber. Only young adult worms can be injected into the chamber. After being trapped, the worms were not allowed to escape from the chamber due to limited size of the gap between the wedge structures (Fig. 3b). Once the target worms were injected into the chamber, the corresponding micro-valve was closed by compressed $\mathrm{N}_{2}(100-300 \mathrm{kPa})$. By opening and closing the microvalves, well-defined numbers of the worms were all placed into the four chambers (Fig. 3e). Finally, the micro-valves were all opened for injecting different concentration of bacteria solution to culture the worms.

For imaging of the worms in the culture chamber, the pneumatic immobilizing valve was switched on by increasing the air pressure $(50 \mathrm{kPa})$ in the control layer (Fig. 3c, Video S2). The deformable PDMS membrane was then pushed down to squeeze the worms to the side of the chamber so that the worms could be immobilized. After imaging, the pneumatic valves were switched off by decreasing the air pressure and the worms could be released and recover quickly (Fig. 3d). We periodically immobilized the worms in the chambers over the course of their lifespans for stimulation and imaging.

\subsection{Lifespan assay}

For lifespan assay on chip, the microfluidic devices were first incubated with Pluronic F-127 solution for $30 \mathrm{~min}$ followed by rinsing with M9 buffer. Pluronic solution is a kind of surface-activating agent, which prevents free bacteria from depositing on the surface of the PDMS and inhibits air bubble generated in the channels. Bacteria was suspended in the M9 buffer solution with $5 \%$ Pluronic F-127 at the concentration of $10^{9}$ or $10^{6}$ cells $\mathrm{mL}^{-1}$, which could prevent bacterial proliferation and reduce bacteria accumulation [7]. The synchronized young adult worms were washed off the plates and suspended in M9 buffer. By using the syringe, these worms were loaded into the culture chambers and incubated with different concentration of bacteria solution at $24^{\circ} \mathrm{C}$. The worms were judged as dead when they did not swim in response to the change of flow rate in the microchannels. For lifespan assay on agar plates, young adult worms were transferred to new NGM agar plates with different concentrations of bacterial solution containing $100 \mu \mathrm{g} \mathrm{ml}^{-1}$ ampicillin to inhibit bacterial growth. The worms were judged as dead when they did not move in response to the touch of an eyebrow hair on agar plates. 

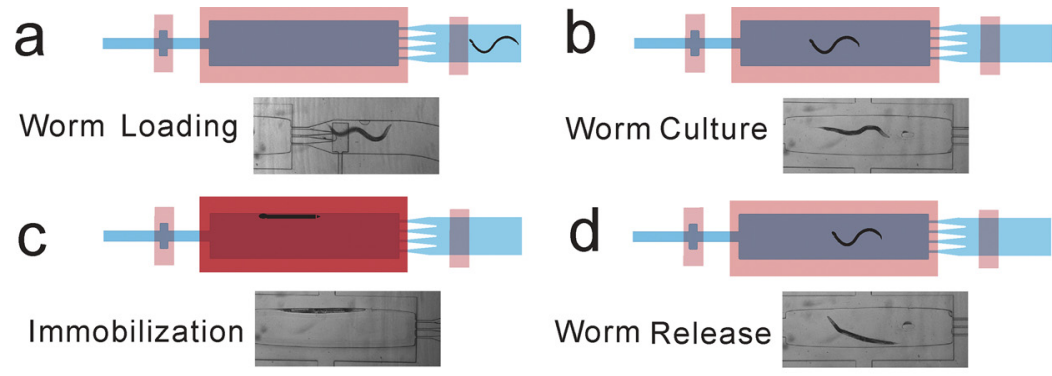

Worm Culture

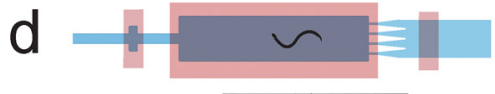

Worm Release
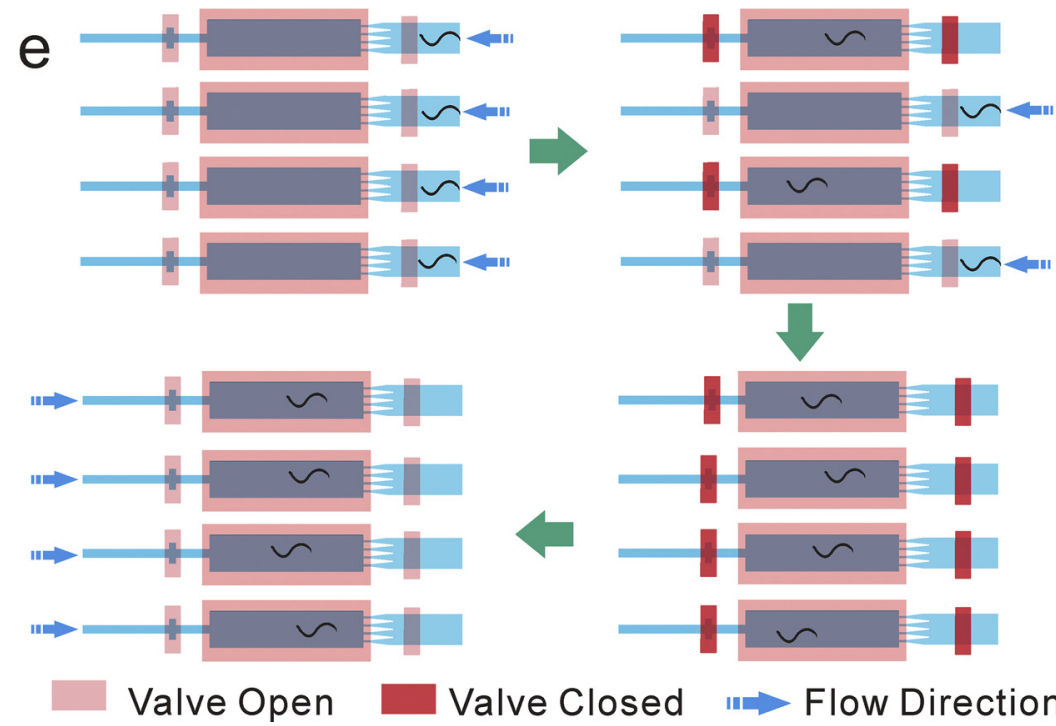

\section{Valve Open}

Valve Closed

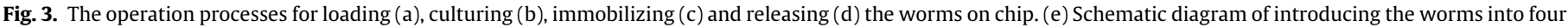
chambers by switching loading pneumatic valves.

The lifespan of each group was calculated according to the survival rate of the trapped worms. The day of loading the worms into the chambers was defined as day 0 . As a comparison, the survival rate of the worms cultured in the NGM agar plates were also monitored every day under the same concentration of bacteria. The statistical significance was determined by Log-rank (Mantel-Cox) tests.

\subsection{Stress response assay}

To investigate stress response of $C$. elegans, the transgenic strain TJ356 were used for imaging analysis of subcellular distribution of DAF-16:GFP fusion protein. Green fluorescence of TJ356 worms was examined by using an inverted fluorescent microscope (IX71, Olympus, Tokyo, Japan) equipped with a CCD camera (QICAM, QImaging, St. Helens, UK) and an EXFO X-Cite 120 series microscope light source (Quebec City, Canada). The image acquisition was processed by the software Image-Pro Plus 6.0 (Media Cybernetics, Bethesda, MD, USA). The emitted light was passed through the GFP cube (excitation, $472 / 30 \mathrm{~nm}$; emission, 520/35 nm; dichroic mirror, $495 \mathrm{~nm}$; Olympus, Japan) and focused onto the immobilized worms in the culture chambers using a $10 \times$ objective (NA $=0.3$ ).

\section{Results and discussion}

\subsection{Design of 10-fold dilution microfluidic device}

The proposed 10-fold dilution micro-device was designed based on the hydrodynamic theory. The structure of the dilution chip had two inlets, distribution network channels, and four chambers for culturing and imaging C. elegans. Two syringe pumps were applied on the inlet module to drive bacteria solution into the distribution channels. The length of the channel determined the mixing ratio of two solutions from the upstream. By accurate calculation of each channels length, output concentration could form 10-fold logarithmic concentration gradient. For fully mixing bacteria stream before introduced into the next dilution step, long helical serpentine channels were employed in this experiment. Finally, liquids with different concentration of bacteria flow were distributed into the culture chamber. The microchannel for loading C. elegans were designed with a width of $25 \mu \mathrm{m}$ as well as some narrow wedge structures at the end of worm inlet channel so that the young adult worms could easily enter into but not escape from the chamber during the experiment. The culture chambers were designed with a dimension of $5 \mathrm{~mm}$ in length and $0.5 \mathrm{~mm}$ in width in order to immobilize the worms through pneumatic valves for imaging analysis while avoid stress effects resulted from confined space.

\subsection{Simulation and fluorescence measurement}

The microfluidic logarithmic gradient generator was designed with the parameters listed in Table 1 . In order to quantify the velocity field and the concentration field in the micro-device at different areas and assess its degree of uniformity, 3-Dimensional model of the micro-device was simulated in COMSOL Multiphysics (COMSOL 3.4 Sweden). The flow pattern and solution mixture of samples were characterized by the incompressible Navier-Stokes equation, convection and diffusion model based on finite element method. Fig. 4 showed the simulation results of generating 10 -fold logarithmic gradients. For the velocity field, the fluidic speeds in chambers 
a

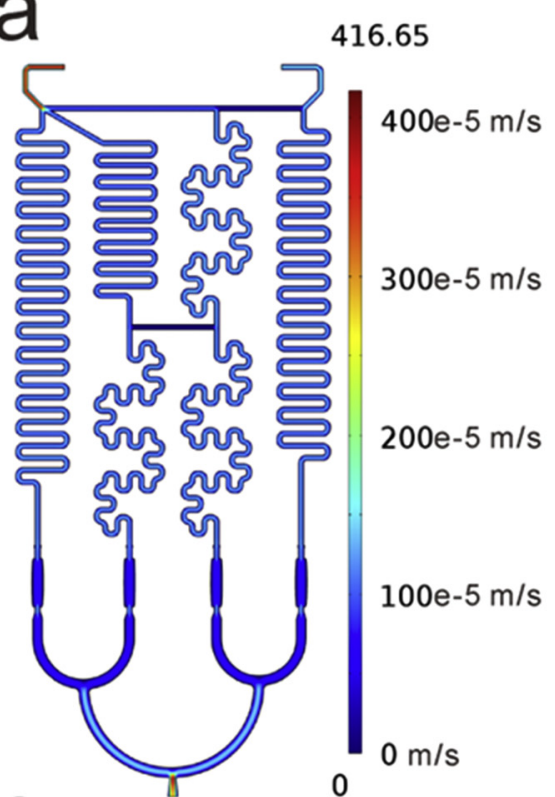

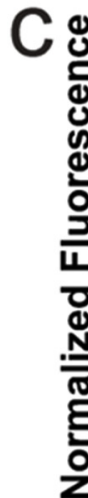

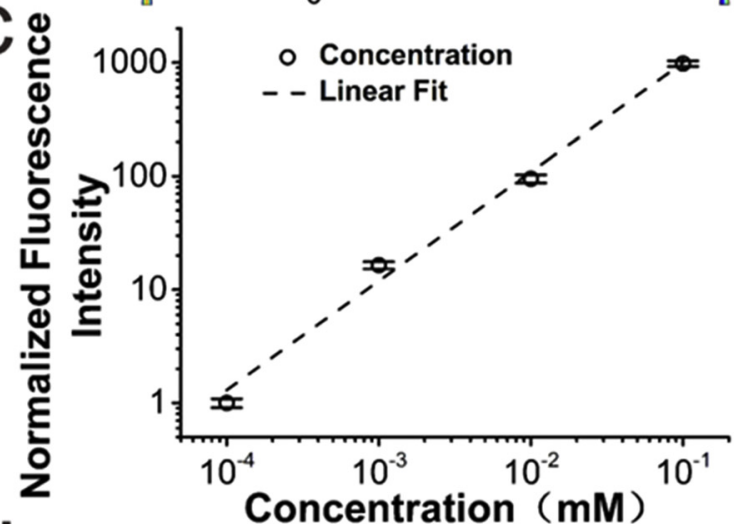

$d$

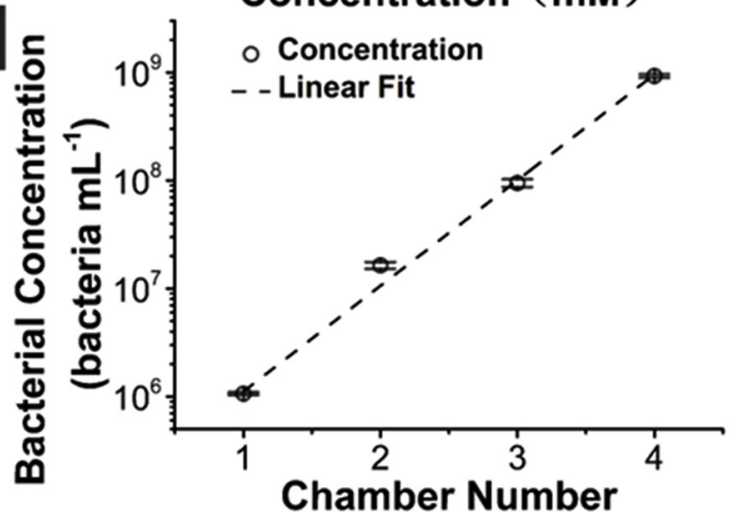

1001.9

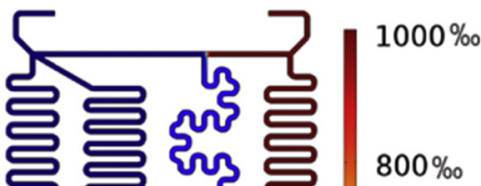

$600 \%$

$400 \%$

$200 \%$

$0 \%$
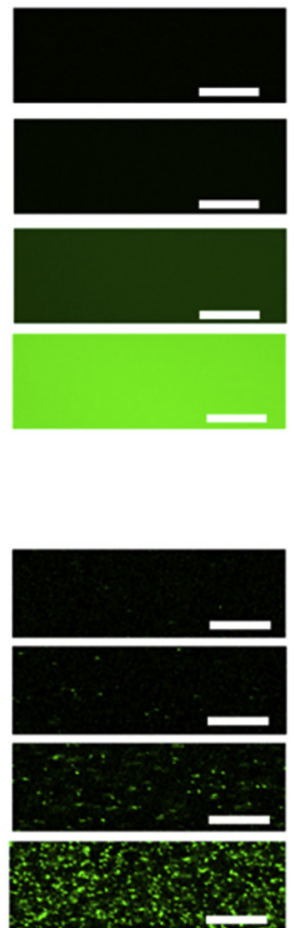

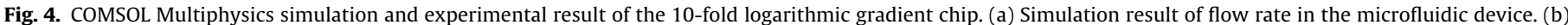

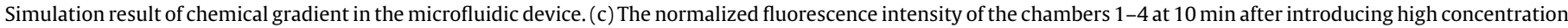

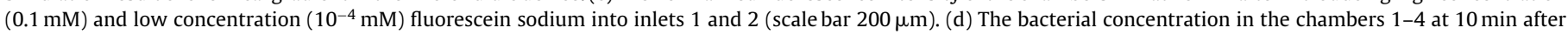
introducing high concentration $\left(10^{9}\right.$ cells $\left.\mathrm{mL}^{-1}\right)$ and low concentration $\left(10^{6}\right.$ cells $\left.\mathrm{mL}^{-1}\right)$ of bacterial suspension into inlets 1 and 2 (scale bar $50 \mu \mathrm{m}$ ).

were the same among four microchannels (Fig. 4a), which provided the necessary amount of conditions used in worms experiment. For the concentration field, the right chamber was almost 10-fold higher than the left one as shown in Fig. 4b.

To further confirm this simulation result, we measured the fluorescence density of fluorescein sodium solution in the chambers. The high concentration of fluorescein sodium salt solution $(0.1 \mathrm{mM})$ was pumped into the inlet 1 with $1 \mu \mathrm{L} \mathrm{min}^{-1}$ and the low concen- tration of solution $\left(10^{-4} \mathrm{mM}\right)$ was pumped into the inlet 2 with $2.6 \mu \mathrm{L} \mathrm{min}^{-1}$. All fluorescence images were captured at the chamber areas. Fig. 4c showed the normalized fluorescence intensities of the diluted solutions in four chamber areas, indicating that the diluted concentrations were $10^{-4} \mathrm{mM}, 10^{-3} \mathrm{mM}, 10^{-2} \mathrm{mM}, 0.1 \mathrm{mM}$ respectively. The experiments were repeated three times, and the correlation coefficient of linear regression on the logarithmic scale was 0.976 , the average standard deviation was \pm 0.055 , which 
confirmed that logarithmic gradient could be obtained across the chambers with high matching degree. The results satisfied the requirements in the following experimental study.

In addition, we directly counted the number of bacteria in the four chambers for evaluating the mixture effect of bacteria. Transgenic E. coli BL21 (DE3) bacteria expressing green fluorescent protein was used. High concentration of $10^{9}$ bacteria $\mathrm{mL}^{-1}$ and low concentration of $10^{6}$ bacteria $\mathrm{mL}^{-1}$ were injected into inlet 1 and 2 , respectively. After $30 \mathrm{~min}$ of generating bacterial concentration gradient, each fluorescence dot represented a single bacteria as shown in Fig. 4d, confirming the generation of logarithmic gradient of bacteria. The correlation coefficient of linear regression on the logarithmic scale was 0.965 , the average standard deviation was \pm 0.05 . Thus, this micro-device could realize worm lifelong culture, bacterial food control, worm immobilization and imaging analysis in real time.

\subsection{Effects of bacterial concentration on the lifespan}

In order to investigate the longevity of the worms in response to various concentrations of bacteria, this microfluidic device was used to generate 10-fold logarithmic concentration gradient from $10^{6}$ to $10^{9}$ bacteria $\mathrm{mL}^{-1}$. Each chamber loaded 10 worms (more worms with increasing the chamber size were allowed if needed). These worms would produce hundreds of eggs during their total lifespan in the worm culture chambers. However, the newborn L1 worms $(<10 \mu \mathrm{m}$ in width) after hatch for about $11 \mathrm{~h}$ would be quickly flushed out of the chamber. In addition, the bottom layer fabricated with the 20:1 mixing ratio of PDMS could be easily deformed under the pressure, allowing the remaining eggs to be flushed out of the chamber. Besides, once we found that individual egg was trapped at the wedge structure, the corresponding micro-valve was slightly deformed by compressed $\mathrm{N}_{2}$ to release the trapped egg. Therefore, the flow profile would not be changed by the eggs clogging. After the formation of stable concentrations of bacteria, the longevity feature of $C$. elegans loaded in the culture chambers was characterized.

As shown in Fig. 5, the wild-type worms maintained in the micro-device with high bacterial density $\left(10^{9}\right.$ bacteria $\left.\mathrm{mL}^{-1}\right)$ had a slightly longer lifespan than that cultured on agar plates seeded with bacterial lawn, suggesting this concentration of bacteria was a suitable living condition for C. elegans. Obviously increased lifespan was found in the worms fed with bacteria dilution solution with the concentration of $10^{8}$ cells $\mathrm{mL}^{-1}$ in comparison with the concentration of $10^{9}$ bacteria $\mathrm{mL}^{-1}$. However, the survival rate was observed to decrease rapidly for the worms fed with $10^{7}$ and $10^{6}$ bacteria $\mathrm{mL}^{-1}$ suspension solution (Fig. 5a). Besides, the survival rate and lifespan of worms cultured on agar plates with a series of bacteria concentration were also investigated (Fig. 5b). Both the survival rate and lifespan of worms fed in the micro-device were almost the same as those of worms cultured on agar plates.

The survival curve, averaged and maximum lifespan of the worms cultured in the micro-device and agar plates were also further measured. The statistical analyses were illustrated in Table S1 and S2. The results showed that the mean and maximum lifespan of the worms cultured in the micro-device were significantly increased to 13.5 day and 22 day respectively under the condition of food restriction $\left(10^{8}\right.$ bacteria $\left.\mathrm{mL}^{-1}\right)$, compared to 11.3 day and 18 day under the condition of enough food $\left(10^{9}\right.$ bacteria $\left.\mathrm{mL}^{-1}\right)$ (Fig. $5 \mathrm{c}$ and Table S1). Meanwhile, the lifespan of the worms under the condition of food absence was also investigated. The mean lifespan was observed to be 7.0 days and 5.6 days, and the maximum lifespan was also reduced to 13 days and 10 days when the worms cultured in the micro-device were fed with $10^{7}$ and $10^{6}$ bacteria $\mathrm{mL}^{-1}$ solution, respectively (Fig. $5 \mathrm{c}$ and Table $\mathrm{S} 1$ ). Both the mean lifespan and maximum lifespan of worms fed in the micro-
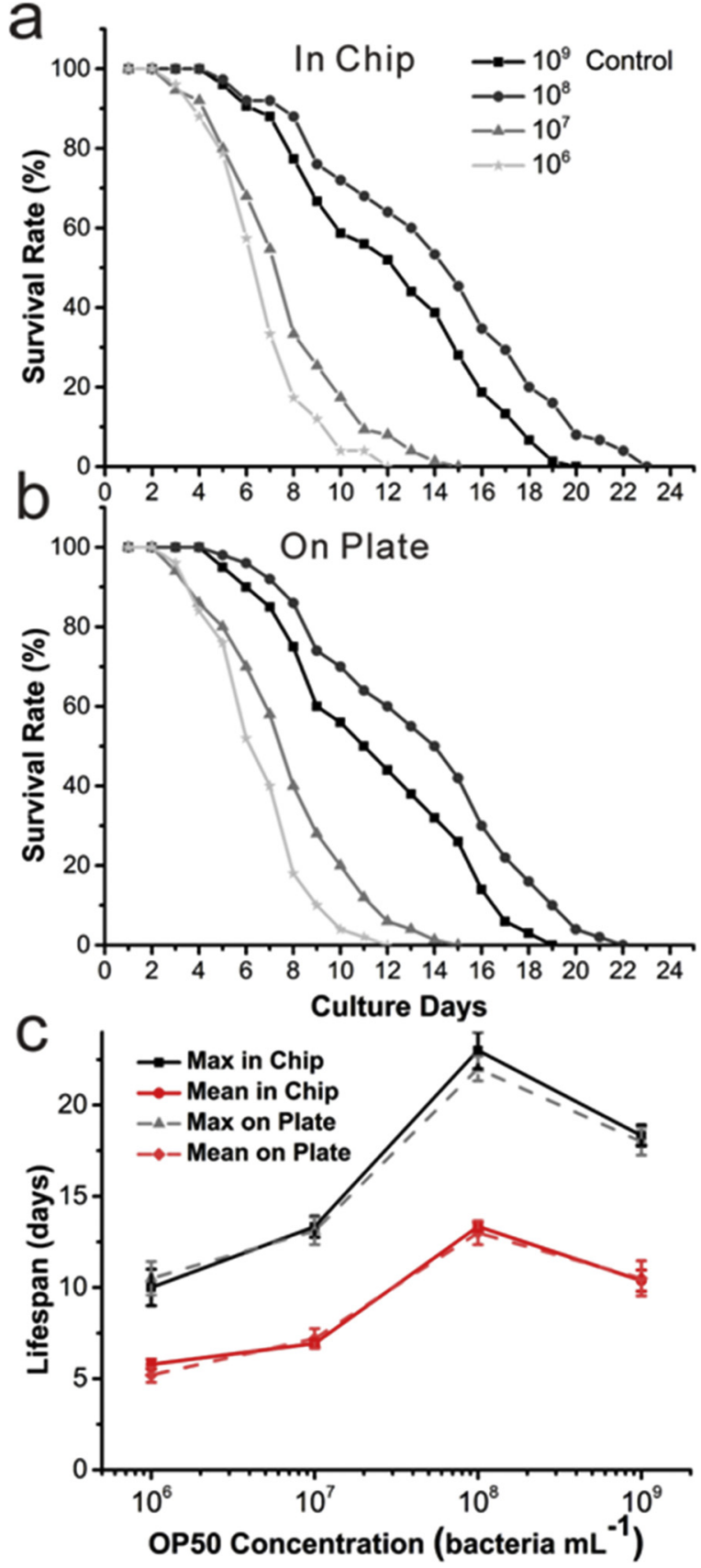

Fig. 5. Effects of bacterial concentrations on survival rate and lifespan of C. elegans. Survival curves of wild-type worms cultured in the chip (a) and on agar plates (b). (c) Comparison of the mean and maximum lifespan of the worms cultured in the chip and on agar plates at the concentrations of from $10^{6}$ to $10^{9}$ bacteria $\mathrm{mL}^{-1}$. $\operatorname{DR}\left(10^{8}\right.$ bacteria $\left.\mathrm{mL}^{-1}\right)$ leads to increase $(\mathrm{p}=0.046)$ in mean lifespan of the worms compared to $10^{9}$ bacteria $\mathrm{mL}^{-1}$ (suitable living condition, set as control). While the mean lifespan was observed to decrease for the worms fed with $10^{7}(\mathrm{p}=0.0007)$ and $10^{6}(\mathrm{p}<0.0001)$ bacteria $\mathrm{mL}^{-1}$ suspension solution compared to $10^{9}$ bacteria $\mathrm{mL}^{-1}$. Data are shown as the mean $\pm S E M . N=3$ of 10 animals scored per experiment. The statistical significance was determined by Log-rank (Mantel-Cox) tests.

device were almost the same as those of worms cultured on agar plates (Fig. 5c and Table S2). These results indicated that the lifespan extension of the worms was induced by DR as bacterial food density was reduced to the optimal dilution of $10^{8}$ bacteria $\mathrm{mL}^{-1}$. Further dilutions of bacteria food would cause reduced mean and maximum lifespan, presumably due to that the food abundances at the concentration of less than $10^{7}$ bacteria $\mathrm{mL}^{-1}$ could not fulfill the worms' basic physiological needs. Taken together, above results 
indicated that our microfluidic method could be used for analyzing the effects of DR on the worms.

\subsection{Stress response to different concentration of bacteria}

To investigate the mechanism of the action of food restriction in delaying aging, we further analyzed the effects of DR on other compounds that can regulate the lifespan of C. elegans. DAF-16, one of the members of the FOXO transcription factor that acts in dauer formation, longevity, fat metabolism, stress response, and innate immunity $[42,43]$. In live C. elegans under standard culture condition, the DAF-16 protein is broadly expressed both in the cytoplasm and nuclei. Several known regulators of DAF-16, including the insulin receptor mutant or starvation, could trigger the nuclear localization of DAF-16 to extend the lifespan of the worm.

Here we investigated whether food absence could induce the nuclear localization of DAF-16 using this microfluidic device. The strain of TJ356 expressing DAF-16:GFP was used to fluorescently observe the localization of DAF-16 for monitoring the physiological stress response of $C$. elegans to different concentration of bacteria. When the TJ356 worms were loaded into the micro-device, the fluorescence signal of DAF-16:GFP first appeared diffused in the whole cells of the worms. After $30 \mathrm{~min}$ of generation of bacteria concentration gradient, the spatial distribution of DAF-16 changed considerably in the worms fed with bacteria dilution at the concentration of $10^{6}$ and $10^{7}$ cells $\mathrm{mL}^{-1}$. Compared to the effect of starvation, food restriction $\left(10^{8}\right.$ bacteria $\left.\mathrm{mL}^{-1}\right)$ weakly stimulated DAF-16 translocation to the nucleus (Fig. 6a). Meanwhile, the subcellular distribution of DAF-16:GFP of the worms cultured on the agar plates were also observed under the same condition. In order to easily observe and compare the imaging results, the worms cultured on agar plates were immobilized by another microfluidic chip containing a pneumatic valve previously designed by us [38]. There was no difference in DAF-16:GFP subcellular distribution in the worms cultured in the micro-device and on agar plates (a).

We further quantitatively investigated the sustain time of nuclear localization of DAF-16:GFP in the worms cultured with different concentration of bacteria by using the micro-device. As illustrated in Fig. $6 \mathrm{~b}$, when food is abundant $\left(10^{9}\right.$ bacteria $\left.\mathrm{mL}^{-1}\right)$, no nuclear localization of DAF-16 was observed as the same level as that cultured on standard NGM agar plates. Under the condition of food absence $\left(10^{6}-10^{8}\right.$ bacteria $\left.\mathrm{mL}^{-1}\right)$, the worms all displayed progressive DAF-16:GFP nuclear localization. However, the nuclear localization of DAF-16 was shown to be evident after $20 \mathrm{~min}$ and grown up quickly as time goes by in response to $10^{6}$ and $10^{7}$ bacteria $\mathrm{mL}^{-1}$. The nuclear localization of DAF-16 last for a long time of $80 \mathrm{~min}$, and its number was as high as about 60 per worm. Although the worms cultured in the chamber with $10^{8}$ bacteria $\mathrm{mL}^{-1}$ appeared similar trend of DAF-16 nuclear localization, the maximum number of nuclei with visible DAF-16:GFP was decreased to 20 per worm, and the duration decreased to $40 \mathrm{~min}$. These results suggested that $C$. elegans responded to DR both in development time and sustain time by showing number of nuclei. Taken together, our proposed microfluidic device was confirmed to be effective for studying bacterial food concentration's effect on the lifespan in C. elegans in a flexible and simple operation format.

\section{Conclusions}

In this work, we developed a microfluidic device to generate logarithmic bacterial concentration gradient to investigate the physiological processes of $C$. elegans. Bacterial food supplements with four orders of magnitude were automatically generated by a serial dilution in the channel network. We employed our platform to evaluate the effects of bacteria food on the lifespan of $C$. ele-
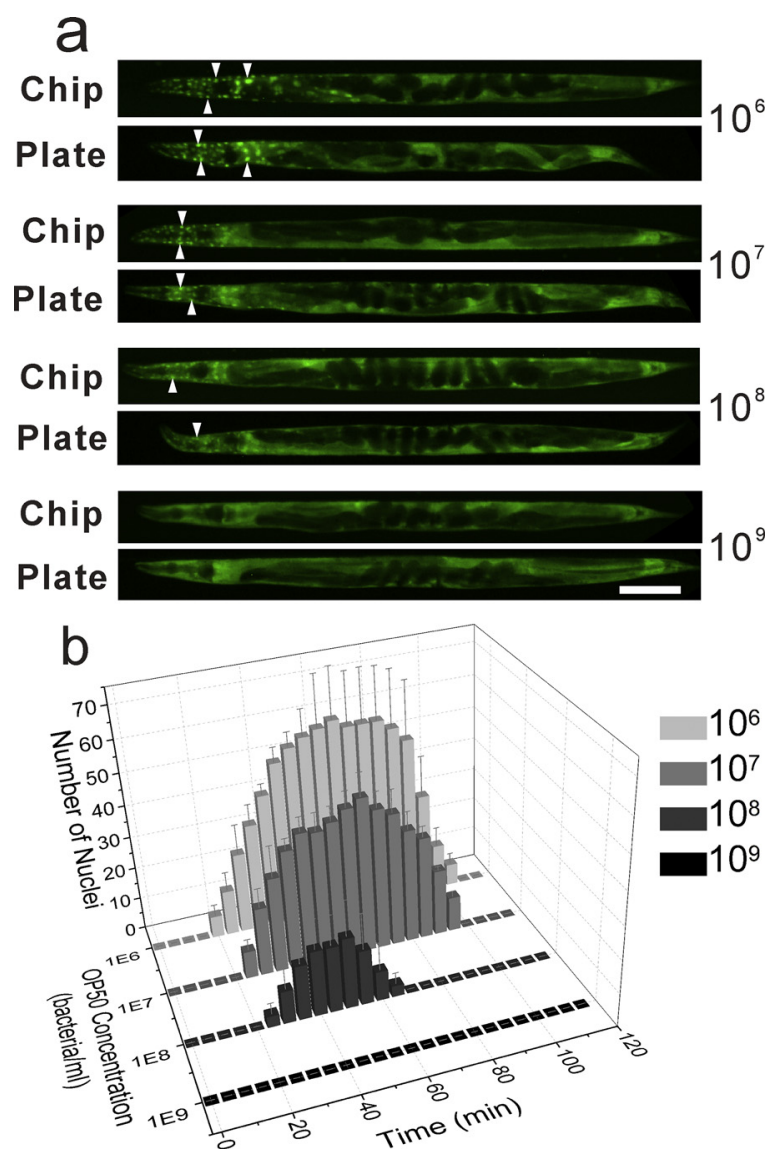

Fig. 6. Kinetics of DAF-16:GFP nuclear localization in wild-type worms in response to different concentration of food. (a) Typical diffusive pattern of DAF-16:GFP in the worms cultured in the chip and on agar plates for $30 \mathrm{~min}$ at the concentrations of from $10^{6}$ to $10^{9}$ bacteria $\mathrm{mL}^{-1}$. Arrowheads indicate nuclear localization (scale bar $100 \mu \mathrm{m}$ ). (b) Effects of food on nuclear DAF-16 localization in the worms cultured in the chip at different concentration of bacteria. Nuclear localization is defined as nuclei-size spots that are significantly brighter than background. The pictures are captured every five minutes. Error bar represents the SEM of five experiments.

gans in a wide range of concentration. The results showed that $10^{8}$ bacteria/mL could extend the lifespan of $C$. elegans and further dilutions would reduce the lifespan, which proposed a new DR method in C. elegans by on-chip culturing the worms in constant bacterial food dilution at low nutrient levels. We further investigated the stress response of $C$. elegans to various concentration of bacterial food for studying the mechanism of DR's effect. Imaging analysis showed that DR regulated the subcellular localization DAF-16 in the worms, the duration and number of which were different from that induced by starvation.

Thus, this developed micro-device offered advantages for studying lifespan extension by bacteria concentration in C. elegans. It had several unique advantages including flexible manipulation capacity to create various concentration gradients, short consuming time to establish gradient and high reproducibility. The microfluidic nature of the micro-device makes it a useful tool for exposing the worms to complex environment. Researchers can employ this microdevice to investigate the dynamics of worms infection caused by pathogenic bacteria in a wide range of concentration. This microdevice can be used to stimulate the trapped worms with temporal concentration gradients or pulses of dissolved drugs or chemicals. It can be applied to identify antimicrobial activity of antibiotics and worms' environmental adaptation. In addition, the microfluidic chip enabled real-time imaging data from the treated worms, which 
could be used for analysis of dynamical behavior in the worms at the physiological and molecular level.

\section{Acknowledgements}

We gratefully acknowledge the financial supports from National Natural Science Foundation of China (21475049, 31471257 and 21275060) and National Key R\&D Program of China (2016YFF0100801). All the worm strains used in this study were provided by the Caenorhabditis Genetics Centre (CGC), which is funded by the NIH National Centre for Research Resources.

\section{Appendix A. Supplementary data}

Supplementary data associated with this article can be found, in the online version, at http://dx.doi.org/10.1016/j.snb.2017.08.088.

\section{References}

[1] M. Hansen, A. Chandra, L.L. Mitic, B. Onken, M. Driscoll, C. Kenyon, A role for autophagy in the extension of lifespan by dietary restriction in C. elegans, PLoS Genet. 4 (2008) e24.

[2] E.L. Greer, A. Brunet, Different dietary restriction regimens extend lifespan by both independent and overlapping genetic pathways in C. elegans, Aging Cell 8 (2009) 113-127.

[3] E.L. Greer, D. Dowlatshahi, M.R. Banko, J. Villen, K. Hoang, D. Blanchard, S.P. Gygi, A. Brunet, An AMPK-FOXO pathway mediates longevity induced by a novel method of dietary restriction in C. elegans, Curr. Biol. 17 (2007) 1646-1656

[4] N.A. Bishop, L. Guarente, Two neurons mediate diet-restriction-induced longevity in C. elegans, Nature 447 (2007) 545-549.

[5] S.K. Park, C.D. Link, T.E. Johnson, Life-span extension by dietary restriction is mediated by NLP-7 signaling and coelomocyte endocytosis in C. elegans, FASEB J. 24 (2010) 383-392.

[6] S. Brenner, The genetics of Caenorhabditis elegans, Genetics 77 (1974) 71-94.

[7] B. Xian, J. Shen, W. Chen, N. Sun, N. Qiao, D. Jiang, T. Yu, Y. Men, Z. Han, Y. Pang, M. Kaeberlein, Y. Huang, J.D. Han, WormFarm: a quantitative control and measurement device towards automated $C$ elegans aging analysis, Aging Cell 12 (2013) 398-409.

[8] C.J. Kenyon, The genetics of ageing, Nature 464 (2010) 504-512.

[9] S.E. Hulme, S.S. Shevkoplyas, A.P. McGuigan, J. Apfeld, W. Fontana, G.M. Whitesides, Lifespan-on-a-chip: microfluidic chambers for performing lifelong observation of C. elegans, Lab Chip 10 (2010) 589-597.

[10] J. Krajniak, H. Lu, Long-term high-resolution imaging and culture of C. elegans in chip-gel hybrid microfluidic device for developmental studies, Lab Chip 10 (2010) 1862-1868

[11] B. Lakowski, S. Hekimi, The genetics of caloric restriction in Caenorhabditis elegans, Proc. Natl. Acad. Sci. U. S. A. 95 (1998) 13091-13096.

[12] R. Curtis, G. O'Connor, P.S. DiStefano, Aging networks in Caenorhabditis elegans: AMP-activated protein kinase (aak-2) links multiple aging and metabolism pathways, Aging Cell 5 (2006) 119-126.

[13] S. Kim, H.J. Kim, N.L. Jeon, Biological applications of microfluidic gradient devices, Integr. Biol. 2 (2010) 584-603.

[14] S.E. Hulme, G.M. Whitesides, Chemistry and the worm: Caenorhabditis elegans as a platform for integrating chemical and biological research, Angew. Chem. Int. Ed. Engl. 50 (2011) 4774-4807.

[15] N.A. Bakhtina, J.G. Korvink, Microfluidic laboratories for C. elegans enhance fundamental studies in biology, RSC Adv. 4 (2014) 4691-4709.

[16] Y. Li, X. Feng, W. Du, Y. Li, B.-F. Liu, Ultrahigh-throughput approach for analyzing single-cell genomic damage with an agarose-based microfluidic comet array, Anal. Chem. 85 (2013) 4066-4073.

[17] Y. Li, X. Yan, X. Feng, J. Wang, W. Du, Y. Wang, P. Chen, L. Xiong, B.-F. Liu, Agarose-based microfluidic device for point-of-care concentration and detection of pathogen, Anal. Chem. 86 (2014) 10653-10659.

[18] D.R. Albrecht, C.I. Bargmann, High-content behavioral analysis of Caenorhabditis elegans in precise spatiotemporal chemical environments, Nat. Methods 8 (2011) 599-605.

[19] K. Chung, M. Zhan, J. Srinivasan, P.W. Sternberg, E. Gong, F.C. Schroeder, H. Lu, Microfluidic chamber arrays for whole-organism behavior-based chemical screening, Lab Chip 11 (2011) 3689-3697.

[20] H. Wen, X. Gao, J. Qin, Probing the anti-aging role of polydatin in Caenorhabditis elegans on a chip, Integr. Biol. 6 (2014) 35-43.

[21] M. Cornaglia, L. Mouchiroud, A. Marette, S. Narasimhan, T. Lehnert, V. Jovaisaite, J. Auwerx, M.A.M. Gijs, An automated microfluidic platform for $C$. elegans embryo arraying, phenotyping, and long-term live imaging, Sci. Rep. 5 (2015) 10192

[22] L. Hu, J. Ye, H. Tan, A. Ge, L. Tang, X. Feng, W. Du, B.-F. Liu, Quantitative analysis of Caenorhabditis elegans chemotaxis using a microfluidic device, Anal. Chim. Acta 887 (2015) 155-162.
[23] X.X. Wang, R. Hu, A.L. Ge, L. Hu, S.S. Wang, X.J. Feng, W. Du, B.F. Liu, Highly efficient microfluidic sorting device for synchronizing developmental stages of C. elegans based on deflecting electrotaxis, Lab Chip 15 (2015) 2513-2521.

[24] N. Chronis, M. Zimmer, C.I. Bargmann, Microfluidics for in vivo imaging of neuronal and behavioral activity in Caenorhabditis elegans, Nat. Methods 4 (2007) 727-731.

[25] C.B. Rohde, F. Zeng, R. Gonzalez-Rubio, M. Angel, M.F. Yanik, Microfluidic system for on-chip high-throughput whole-animal sorting and screening at subcellular resolution, Proc. Natl. Acad. Sci. U. S. A. 104 (2007) 13891-13895.

[26] K. Chung, M.M. Crane, H. Lu, Automated on-chip rapid microscopy, phenotyping and sorting of C. elegans, Nat. Methods 5 (2008) 637-643.

[27] S.X. Guo, F. Bourgeois, T. Chokshi, N.J. Durr, M.A. Hilliard, N. Chronis, A. Ben-Yakar, Femtosecond laser nanoaxotomy lab-on-a-chip for in vivo nerve regeneration studies, Nat. Methods 5 (2008) 531-533.

[28] Y. Li, D. Chen, Y. Zhang, C. Liu, P. Chen, Y. Wang, X. Feng, W. Du, B.-F. Liu, High-throughput single cell multidrug resistance analysis with multifunctional gradients-customizing microfluidic device, Sens. Actuators B-Chem. 225 (2016) 563-571.

[29] W. Saadi, S.-J. Wang, F. Lin, N. Jeon, A parallel-gradient microfluidic chamber for quantitative analysis of breast cancer cell chemotaxis, Biomed. Microdevices 8 (2006) 109-118.

[30] K. Campbell, A. Groisman, Generation of complex concentration profiles in microchannels in a logarithmically small number of steps, Lab Chip 7 (2007) 264-272.

[31] K. Kirchhof, A. Andar, H.B.c Yin, N. Gadegaard, M.O. Riehle, T. Groth, Polyelectrolyte multilayers generated in a microfluidic device with $\mathrm{pH}$ gradients direct adhesion and movement of cells, Lab Chip 11 (2011) 3326-3335.

[32] K.W. Oh, K. Lee, B. Ahn, E.P. Furlani, Design of pressure-driven microfluidic networks using electric circuit analogy, Lab Chip 12 (2012) 515-545.

[33] K. Lee, C. Kim, B. Ahn, R. Panchapakesan, A.R. Full, L. Nordee, J.Y. Kang, K.W. Oh, Generalized serial dilution module for monotonic and arbitrary microfluidic gradient generators, Lab Chip 9 (2009) 709-717.

[34] C. Kim, K. Lee, J.H. Kim, K.S. Shin, K.-J. Lee, T.S. Kim, J.Y. Kang, A serial dilution microfluidic device using a ladder network generating logarithmic or linear concentrations, Lab Chip 8 (2008) 473-479.

[35] Y. Li, P. Chen, Y. Wang, S. Yan, X. Feng, W. Du, S.A. Koehler, U. Demirci, B.F. Liu, Rapid assembly of heterogeneous 3D cell microenvironments in a microgel array, Adv. Mater. 28 (2016) 3543-3548.

[36] Y. Li, X. Feng, Y. Wang, W. Du, P. Chen, C. Liu, B.-F. Liu, Assembly of multiple cell gradients directed by three-dimensional microfluidic channels, Lab Chip 15 (2015) 3203-3210.

[37] J. Yang, Z. Chen, P. Ching, Q. Shi, X. Li, An integrated microfluidic platform for evaluating in vivo antimicrobial activity of natural compounds using a whole-animal infection model, Lab Chip 13 (2013) 3373-3382.

[38] Y. Wang, J. Wang, W. Du, X.J. Feng, B.-F. Liu, Identification of the neuronal effects of ethanol on C. elegans by in vivo fluorescence imaging on a microfluidic chip, Anal. Bioanal. Chem. 399 (2011) 3475-3481.

[39] L. Hu, A. Ge, X. Wang, S. Wang, Y. Gao, X. Feng, W. Du, B.-F. Liu, An on-demand gas segmented flow generator with high spatiotemporal resolution for in vivo analysis of neuronal response in C. elegans, Lab Chip 16 (2016) 4020-4027.

[40] X. Ai, W. Zhuo, Q. Liang, P.T. McGrath, H. Lu, A high-throughput device for size based separation of C. elegans developmental stages, Lab Chip 14 (2014) $1746-1752$.

[41] H. Wen, W. Shi, J. Qin, Multiparameter evaluation of the longevity in C. elegans under stress using an integrated microfluidic device, Biomed. Microdevices 14 (2012) 721-728

[42] X. Wang, L. Tang, Y. Xia, L. Hu, X. Feng, W. Du, B.-F. Liu, Stress response of Caenorhabditis elegans induced by space crowding in a micro-column array chip, Integr. Biol. 5 (2013) 728-737.

[43] R. Kopito, E. Levine, Durable spatiotemporal surveillance of Caenorhabditis elegans response to environmental cues, Lab Chip 14 (2013) 764-770.

\section{Biographies}

Anle Ge is a Ph.D. candidate in Huazhong University of Science and Technology (HUST), China. His main research interest focuses on microfluidic-based methods for C. elegans investigation.

Liang Hu received his Ph.D. degree from HUST. His main research interest focuses on microfluidic-based methods for analyzing C. elegans.

Xixian Wang received his Ph.D. degree from HUST. His main research interest is the development of microfluidic platform for studying C. elegans.

Jinchi Zhu is a Ph.D. candidate in HUST. His research interest focuses on microfluidicbased methods for biological applications and bioengineering.

Xiaojun Feng received his Ph.D. degree in Biology from the University at Albany, State University of New York in 2006. Thereafter, he moved to HUST, China, continuing his postdoctoral research in the College of Life Science \& Technology. He is currently an associate professor at HUST. His research interest includes BioMEMS, microfluidics, point-of-care testing and mass spectrometry-based proteomics. 
Wei Du received his Ph.D. degree in Biology from Wuhan University, China in 2005. He is currently an associate professor at HUST. His main research interest focuses on the development of microfluidic analysis of C. elegans.

Bi-Feng Liu received his Ph.D. degree in Analytical Chemistry from Wuhan University (China) in 1999 and continued his postdoctoral research in the College of Life
Science. From 2001, he moved to Japan and worked in Prof. Terabe's group (Japan) as a JSPS postdoctoral fellow. He joined HUST as a professor in 2003. He is now serving as deputy Dean of the College of Life Science \& Technology. His research focuses on systems biology-oriented analytical science in the areas of mass spectrometry coupled micro-separation, microfluidics and molecular imaging. 